Ketelhut, Jörn. Der EuGH und die deutschen Arbeitsgerichte - Strategische Interaktionen in komplexen Entscheidungskontexten. Eine politikwissenschaftliche Analyse judizieller Governance im EU-Mehrebenensystem. Baden-Baden. Nomos 2010. 264 Seiten. $59 €$.

Jörn Ketelhut hat ein Buch vorgelegt, das gleich in mehrfacher Hinsicht als außerordentlich innovativ zu würdigen ist. Erstens leistet es einen wichtigen Beitrag zu dem in Deutschland nach wie vor stark vernachlässigten Forschungsgebiet der Rolle von Gerichten im politischen Prozess. Gerichte werden dabei als Akteure in komplexen Governance-Arrangements untersucht. Zweitens stellt Ketelhut das Handeln von Gerichten in einem judiziellen Mehrebenensystem ins Zentrum der Betrachtung. Er untersucht einen grundlegenden Wirkungsmechanismus der „Integration durch Recht“, nämlich die Umsetzung von Entscheidungen des Gerichtshofs der Europäischen Union $(\mathrm{EuGH})$ durch die deutschen Gerichte. Diese Perspektive wird in der anglo-amerikanischen Forschung vor allem mit dem Namen Karen Alter verknüpft, ist in der deutschen Debatte jedoch bisher so gut wie nicht präsent. Im Kontext seiner Mehrebenenanalyse nimmt Ketelhut drittens auch die Rolle der deutschen Fachgerichte (hier: der Arbeitsgerichte) in den Blick, während sich der allergrößte Teil der bestehenden Literatur ausschließlich am EuGH oder an den Verfassungsgerichten abarbeitet. Und viertens verfolgt Ketelhut analytisch eine Perspektive, die in einem ohnehin kleinen Forschungsgebiet eher selten anzutreffen ist. Nicht der vermeintliche Median-Richter und seine politischen Policy-Präferenzen ste- hen im Zentrum der Betrachtung, sondern die inhaltliche Dimension des Rechts, wodurch auch zahlreiche Anknüpfungspunkte zur Rechtswissenschaft bestehen. Rechtsentwicklung wird als ein diskursiver Quasi-Verhandlungsprozess begriffen, in dem „Forderungen, Argumente und Angebote" (170) ausgetauscht werden.

Das Kernargument lautet, bei der „Integration durch Recht" handele es sich nicht um einen „sachlogischen Prozess“, der einer „expansiven Evolutionsdynamik" unterliege (241). Der vom EuGH proklamierte Vorrang des Gemeinschaftsrechts bedeute in der Praxis keinesfalls, dass die europäischen Urteile von den deutschen Fachgerichten lediglich umgesetzt würden. Vielmehr zeichnet Ketelhut anhand verschiedener Fallbeispiele nach, wie die Arbeitsgerichte vor allem das Instrument des Vorabentscheidungsverfahrens systematisch nutzen, um strittige Rechtsfragen (und vermeintlich inakzeptable Lösungsvorschläge des EuGH) immer wieder an die europäische Ebene zu delegieren. Die beiden Ebenen des Rechtssystems treten dadurch in einen verhandlungsähnlichen Prozess ein, in dem über die Angemessenheit rechtlicher Interpretationen und Problemlösungen diskutiert wird. Die Positionen nähern sich schrittweise wechselseitig an. Der zentrale Governance-Mechanismus ist demnach also nicht Hierarchie, sondern Verhandlung.

Die Analyse von Ketelhut leistet einen sehr wichtigen Beitrag zu wissenschaftlich und politisch kontrovers geführten Debatten in der Integrationsforschung und in der politischen Ökonomie. So kann sie zum Beispiel die Auseinandersetzungen über das Zusammenspiel zwischen EuGH und Arbeitsgerichtsbarkeit im Bereich des Arbeitskampf- 
rechts gut informieren. Die in der polit-ökonomischen Literatur diskutierte These, die europäische Rechtsprechung wirke als ein quasi unausweichlicher Mechanismus zur Liberalisierung der koordinierten Ökonomien, dem kein nationaler Akteur etwas entgegensetzen könne, wird durch die Analyse Ketelhuts erheblich in Zweifel gezogen. Die nationale Rechtsprechung wirkt nicht zwingend als Agent des europäischen Prinzipals. Vor diesem Hintergrund kann man dem Buch eine breite Rezeption nur wünschen, weil es die Debatte enorm befruchten könnte.

Freilich stellt sich die Frage, ob die Kommunikation über Gerichtsurteile beziehungsweise über das Vorabentscheidungsverfahren bereits als Verhandlung bezeichnet werden sollte. Vielleicht ist der Interaktionsmodus mit „wechselseitiger Anpassung " treffender charakterisiert. Jedenfalls drängt sich sofort die interessante Anschlussfrage auf, ob es zwischen den verschiedenen gerichtlichen Instanzen auch reale Austauschprozesse „hinter den Kulissen“ gibt, die möglicherweise eine Rolle spielen, in dem Buch jedoch nicht beleuchtet werden.

Und hier liegt ein wahrnehmbares Defizit von Ketelhuts Arbeit. Von einer politikwissenschaftlichen Analyse judizieller Governance im EU-Mehrebenensystem, die im Untertitel angekündigt wird, würde ich mir wünschen, dass sie die strittigen Fälle und die Interaktionsprozesse zwischen den gerichtlichen Instanzen stärker im Politikfeld und in den politischen Konflikten, die diese Fälle hervorgebracht haben, verortet. Dies würde auch die Komplexität der Entscheidungskontexte, in denen sich die Gerichte bewegen, deutlicher hervortreten lassen. Zwar werden die rechtsdogmatischen Interaktionspro- zesse von Ketelhut schlüssig (und übrigens auch für Nicht-Juristen sehr gut lesbar!) dargestellt. Doch die politische Brisanz hinter den einzelnen Rechtsfragen, die Interessenpositionen und -politiken, die außerhalb der rechtlichen Arena mit diesen Urteilen verknüpft werden und die in diese vielleicht auch hineinwirken, bleiben häufig unterbelichtet, obwohl sowohl der EuGH, als auch die Arbeitsgerichte an manchen Stellen explizit politisch argumentieren. Eine politikwissenschaftliche Analyse des Rechts könnte hier noch mehr leisten. Das mindert allerdings nicht den Wert des Buches. Vielmehr werden neue Forschungsperspektiven sichtbar, die diese Variante der politikwissenschaftlichen Befassung mit Gerichtsentscheidungen zu einem attraktiven und zukunftsträchtigen Forschungsfeld werden lassen.

Britta Rehder

\section{METHODEN DER POLITIKWISSENSCHAFT}

\section{Mutz, Diana C. Population-Based \\ Survey Experiments. Princeton, NJ. \\ Princeton University Press 2011. 177 \\ Seiten. 19,99€.}

Die Idee, Experimente im Rahmen von repräsentativen Bevölkerungsumfragen durchzuführen, hat eine lange Tradition innerhalb der methodischen Grundlagenforschung. Allerdings werden in den letzten Jahrzehnten immer häufiger experimentelle Designs in Umfragen eingebettet, um auch substantielle Fragestellungen zu erforschen, wobei der Schwerpunkt bislang vor allem auf der Wahl- und Einstellungsforschung liegt. Abgesehen von einigen Aufsätzen und gelegentlichen Handbuchartikeln wur- 